The Heterocyclic Derivatives of Phosphorus, Arsenic, Antimony, Bismuth and Silicon

By Frederick George Mann. (The Chemistry of Heterocyclic Compounds Series.) Pp. ix +180. (New York and London: Interscience Publishers, Inc., 1950.) $42 s$.

THIS monograph is the first to appear of a series planned to cover the whole field of heterocyclic chemistry. Its scope is limited to a few rare classes of substance, and this has made it possible to present the facts in considerable detail within the compass of a small volume.

All types of cyclic compound having phosphorus, arsenic, antimony, bismuth or silicon as ring members are reviewed, but the arsenic derivatives, having been most studied, occupy two-thirds of the book. The arsenic analogues of most of the familiar polycyclic nitrogen ring systems have been prepared and are here described. The requirements of chemical warfare during and since the First World War no doubt gave an impetus to the study of arsenic compounds, and the sections on phenarsazine chloride and on the products of anti-lewisite reagents are noteworthy from this point of view.

Although this is a highly specialized book, it contains much that is of general chemical interest. In particular, a number of very interesting stereochemical studies by Mann, Turner and their colleagues are described. These include the preparation of the only known stable optically active quaternary phosphonium and arsonium salts and of an active spiroarsonium salt; optically active phenoxarsine and phenoxstibine derivatives which owe their dissymmetry to molecular structures folded across the middle ring; and some special cases of cis-trans isomerism in palladium complexes and in dihydroarsanthrene derivatives.

The book is excellently produced and is generally free from errors; but a confusion has been noted in the numbering of formulæ on pp. 71-72. G. M. B.

\section{Atmospheric Turbulence}

By Prof. O. G. Sutton. (Methuen's Monographs on Physical Subjects.) Pp. viii +108 . (London : Methuen and Co., Ltd., 1949.) 6s. net.

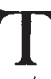
HIS monograph provides a clearly written account of the research which has been carried out during the past twenty years or so, largely in Great Britain and with Prof. O. G. Sutton in the van, on turbulence in the terrestrial boundary layer. The point of view is that of the mathematical physicist, but the mathematical equipment required of the reader is only slight.

Following a general introduction and a concise statement of the observed behaviour of the lower atmosphere (vertical gradients of temperature and wind velocity, eddy velocities and surface shearing stress), the reader is introduced to so-called $K$ theory, an empirical treatment based on the assumption that turbulent diffusion is analogous to molecular diffusion but with an enhanced coefficient of transfer. Its limitations are quickly displayed, and the author then proceeds to show how the Prandtl-von Kármán ideas of the mixing path have been applied with some success to diffusion problems near the ground. Here the author's marriage of mixing-path theory with Taylor's theorem on diffusion by continuous movement is described. A final chapter is devoted to the effects of stable and unstable temperature stratifications and to natural convection.
The subject as presented here is still in the empirical stage. Because of its complexity, the essential physical bonds which must unite it to the basic laws of hydrodynamics and thermodynamics remain to be discovered; and if one has a criticism, it is that the author might have brought out this limitation of his treatment more clearly.

\section{Minerals and How to Study Them}

By the late Edward Salisbury Dana. Revised by Cornelius S. Hurlbut, Jr. (Dana's Series of Mineralogies.) Third edition. Pp. $x+323$. (Now York: John Wiley and Sons, Inc. ; London : Chapman and Hall, Ltd., 1949.) 24s. net.

7 HE fourth and most elementary of the series of text-books by J. D. and E. S. Dana, covering the field of mineralogy, was first published more than half a century ago. This new revision retains essentially the characters of the first edition and remains a well-balanced introduction to the subject. The usual orthodox grouping is followed: chapters on crystallography and the properties of minerals lead to a systematic description of the more commonly occurring mineral species. Finally come short but adequate determinative tables. It is pleasing to find these tables subordinated to the description of the minerals and not, as has sometimes recently been the case, given undue prominence.

In revising the book, Mr. C. S. Hurlbut, jun., has shortened some of the introductory chapters and, in many respects a desirable change, has rearranged the description of the mineral species to follow the chemical classification-oxides, silicates, etc--rather than listing together minerals containing the same elements. The volume is attractively produced and well illustrated with numerous diagrams and photographs. Newcomers to the science will find it a useful and stimulating introduction.

\section{Artificial Radioactivity}

By Prof. P. B. Moon. (Cambridge Monographs on Physics.) Pp. $x+102$. (Cambridge: At the University Press, 1949.) 12s. 6d. net.

$\mathrm{T} T$ is an extremely difficult undertaking to write a 1 hundred pages on the main phenomena and techniques of radioactivity, even if these are limited to the medium and light nuclei, as the author states in the preface. The selection of what can be put of such an enormous field in such a small space must have been one of the main difficulties of the author. In my opinion, this has been solved, and a small book has been created, easily understandable and giving in some places much more than the pure fundamentals.

Identification and measurement of radiations and particles have been condensed into twenty pages, giving, of course, not much more than a very short description of the subjects.

The main emphasis is laid on the two other chapters-on radioactive processes in which the charge of the nucleus is being changed, and such processes where the charge of the nucleus does not change. In a condensed, but very clearly written, version the theories of $\beta$-ray spectra and of the electron-capture process are discussed. A good balance is maintained between theoretical considerations and experimental methods and results.

This is a book which should fulfil the aim to give a reader a clear short version of a number of advances in nuclear physics, without obstructing the view by insertion of too many details. HENRY SeLIGMaN 